\title{
Numerical Simulation of Contaminant Release During Sediment Starting in Dynamic Water Environment
}

\author{
Peng-da Cheng, Xin-guang Zhu, Chun Feng, and Xiao-liang Wang
}

\begin{abstract}
In environmental hydrodynamics, the release of contaminant from sediments is one of the main problems. Based on a large number of experimental data provided by water channel experiments, a coupled mechanical model of overlying water, sediment and contaminant is established in this paper. The process of sediment starting and contaminant release is numerically simulated under different velocity conditions of overlying water. The quantitative relationships among velocity, particle volume fraction, contaminant concentration, turbulent kinetic energy and time are obtained by analyzing the relationship between flow field characteristics and contaminant concentration distribution. The results show that contaminant is released rapidly with the suspension of sediment particles and quickly reaches equilibrium concentrations. When the flow field characteristics (Re) change, the contribution of convection and turbulent diffusion to contaminant release process is different. Establishing a quantitative relationship between hydrodynamic conditions and contaminant release can provide support for constructing water pollution model in lake and reservoir areas.
\end{abstract}

Keywords Sediment contaminant $\cdot$ Velocity $\cdot$ Turbulence kinetic energy $\cdot$ Particle volume fraction $\cdot$ Concentration

\footnotetext{
P.da Cheng $\cdot$ X. Zhu $\cdot$ C. Feng

Institute of Mechanics, Chinese Academy of Sciences, Beijing 100190, China

e-mail: pdcheng @imech.ac.cn
}

\author{
X. Zhu \\ School of Engineering Science, University of Chinese Academy of Sciences, Beijing 100049, \\ China \\ X. Wang $(\bowtie)$ \\ Beijing Institute of Technology, Beijing 100081, China \\ e-mail: wangxiaoliang36@bit.edu.cn




\section{Introduction}

The water is considered to be a natural complex composed of water, dissolved matter, suspended matter, aquatic organisms and sediment [1]. When contaminants enter the water, they will deposit in the sediment, gradually enrich and make the sediment becoming a reservoir of contaminants. The hydraulic conditions are more complicated in the estuary area. The endogenous release effect of the contaminated sediment is more and more obvious. The movement of the sediment with the water flow is very complicated and has great randomness, and the contaminants combined with sediment migrate along with the movement of sediment in water body. On the one hand, the movement and transport of suspended sediment in the water directly affects the temporal and spatial distribution of contaminants; on the other hand, sediment becomes an important potential "source" or "sink" of contaminants as sediment deposits into the bed, and the mechanism of "source" and "sink" will change under certain hydrodynamic disturbances and environmental conditions [2-4]. Hydrodynamics is an important physical factor affecting the diffusion and migration of sediments at the sediment-water interface. On the one hand, dynamic flow enhances the diffusion and mixing ability near the sediment-water interface. On the other hand, the shear stress and turbulence intensity of the sediment-water interface flow will cause the re-suspension of polluted sediment, which will cause a large release of contaminants to the water body. Previous studies on contaminants release from sediments have focused on relatively static waters such as lakes, and systematic and in-depth analysis have been conducted. However, there are few studies on the release law of sediment contaminants in hydrodynamic water, and most of them are qualitative research. The lack of quantitative analysis can easily lead to the exaggeration or underestimation of the re-suspension release of sediment contaminants. In order to understand the physical process and influencing factors of re-suspension release of contaminant, a coupled mechanical model considering overlying water-sediment-contaminant is established. The relationship between velocity, turbulent kinetic energy and vertical distribution of sediment or contaminant under different hydrodynamic conditions is studied. The relationship between contaminant releasing flux and flow characteristics (Reynolds number) is obtained, and the effects of convection and turbulent diffusion on contaminant release are obtained.

\section{Problem Formulation and Solution Methods}

\subsection{Governing Equations}

In this paper, suspended sediment is considered a suspension in the overlying watersediment-contaminant model. Based on analysis of many experimental data $[5,6]$, the viscosity of this kind of suspension can be expressed as a function of the particle volume fraction. Therefore, it is considered that suspension (fluid-particle) has a 
single flow continuum with macroscopic properties (such as density and viscosity). The hypothesis of the model is as follows: (1) The density of each phase is approximately constant. (2) Both phases share the same pressure field. (3) The particle relaxation time is short compared to the time scales of the macroscopic flow.

The mixture of density and viscosity are respectively given by,

$$
\begin{gathered}
\rho=(1-\varphi) \rho_{1}+\varphi \rho_{2} \\
\mu=(1-\varphi) \mu_{1}+\varphi \mu_{2}
\end{gathered}
$$

where $\rho_{1,2}$ and $\mu_{1,2}$ are the density and viscosity properties of fluid and particle respectively, $\varphi$ is the particle volume fraction.

Considering that the suspension can be treated as a continuous medium, the flow is governed by incompressible mass and momentum conservation equations, which require that:

$$
\begin{gathered}
\nabla(\rho u)=0 \\
\rho\left(\frac{\partial u}{\partial t}+u \cdot \nabla u\right)=-\nabla p+\nabla \cdot \tau+\rho g
\end{gathered}
$$

Here, $u$ is the velocity field, $\rho$ is the suspension density, $t$ is the time, $p$ is the pressure field, $\tau$ is the sum of the viscous and turbulent stresses, and $g$ is a body force per unit mass. The $\tau$ is written as for a generalized Newtonian fluid, such that $\tau=$ $\mu \gamma$, where $\mu$ is the suspension viscosity and $\gamma=\nabla u+\nabla u^{T}$. In order to close the governing equations, the $k-\varepsilon$ two-equation formulations are adopted, $\sigma_{k}=1.0, \sigma_{\varepsilon}$ $=1.3, C_{\varepsilon 1}=1.44$, and $C_{\varepsilon 2}=1.92$.

The transport equation for the particles in the flow is ruled by,

$$
\frac{\partial \varphi}{\partial t}+u \cdot \nabla \varphi=-\nabla \cdot N_{\varphi}
$$

where $\mathrm{N}_{\varphi}$ is the total diffusive flux of particles.

The transport equation for the contaminant concentration in the flow is ruled by,

$$
\frac{\partial c}{\partial t}+u \cdot \nabla c=-\nabla \cdot N+R
$$

where $c$ is the relative concentration and is a dimensionless value, $N$ is the total diffusive flux of concentration, $R$ is a source and sink expression, and $u$ is the velocity field.

The viscosity of a particle suspension is usually written as a function of the local particle volume fraction [6, 7, 9-11]. In this paper, the MPQ model [8, 9] is adopted for suspension. 


$$
\mu=\mu_{1}\left(1-\frac{\varphi}{\varphi_{m}}\right)
$$

where $\varphi_{m}$ is the maximum packing concentration, which for solid particles is approximately 0.62 .

\subsection{Geometry, Materials, Boundary Conditions and Initial Conditions}

Now, we consider the case of the particle suspensions flow through a water channel under pressure-driven. In our previous experiments, the water channel was $0.25 \mathrm{~m}$ wide and $3.0 \mathrm{~m}$ long, the water depth was set at $0.1 \mathrm{~m}$, the sediment depth was set at $0.08 \mathrm{~m}$. The geometric model used in the numerical simulation is consistent with the experimental size as Fig. 1, and set the left side of water-sediment interface as coordinate origin. In the numerical simulation, the densities of overlying water and sediment are $997 \mathrm{~kg} / \mathrm{m}^{3}$ and $2650 \mathrm{~kg} / \mathrm{m}^{3}$, respectively. The viscosity of the overlying water is set to $1 \times 10^{-3} \mathrm{~Pa} \mathrm{~s}$. The sediment particle size $\left(\mathrm{D}_{50}\right)$ is $0.03 \mathrm{~mm}$ and the water content is $57.5 \%$.

It is also needed to specify boundary condition; we assume that there is no slip at the water channel wall and zero shear stress at the top surface for flow. For a contaminant $(\mathrm{NaCl})$ concentration calculation, the water channel and the top surface are set to no-flux boundary conditions. We use periodic boundary conditions to the left and right boundaries, i.e., equal velocity, equal concentration, equal volume fraction, equal turbulence kinetic energy and equal turbulence dissipation rate.

For the initial conditions, we set the initial pressure to be related to gravity, the initial velocity is set to 0 , the initial particle volume fraction is set to $42.5 \%$, the initial relative concentration of the overlying water is set to 0 , and the initial relative concentration of the sediment is set to 1 .

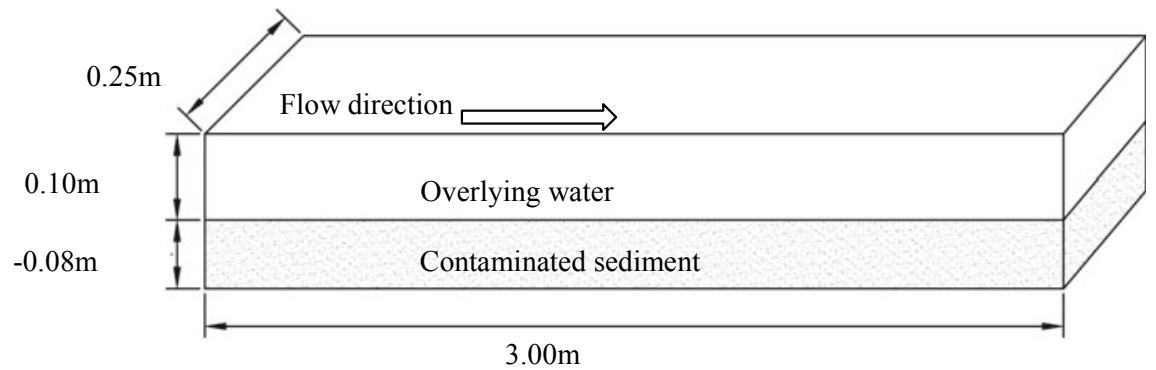

Fig. 1 The geometry of water channel for pressure-driven flow 


\section{Results}

In recent years, the finite-element method (FEM) has developed rapidly and is often used to solve the problem of partial differential equations (PDE) with specific boundary conditions in solid mechanics and fluid mechanics. In this paper, the standard Galerkin finite-element discretization method is used to solve the flow field and contaminant concentration field [12]. In particular, the momentum and turbulence transport equations are dominated by convection and it is well known that a standard Galerkin discretization of such equations leads to oscillations in the solutions. Hence, some form of upwinding is required to suppress these non-physical oscillations. Here, we use a Galerkin least-squares method as described by Hughes et al. $[13,14]$. The current study uses a structured grid system of 82,800 cells, which is chosen after a series of grid independence tests.

Under different hydrodynamic conditions, the movement of sediment with water flow is very complicated and has great randomness, and the contaminant will migrate with the movement of sediment in the water. For quantitative analysis, we introduce the concept of average velocity $(U)$, average volume fraction $(\Phi)$, relative average concentration $(C)$ and average turbulence kinetic energy $(K)$ as follows:

$$
C=\frac{\int_{0}^{\infty} u c d s}{U H} ; \quad \Phi=\frac{\int_{0}^{\infty} u \varphi d s}{U H} ; \quad U=\frac{\int_{0}^{\infty} u^{2} d s}{\int_{0}^{\infty} u d z} ; \quad K=\frac{\int_{0}^{\infty} u k d s}{U H} ;
$$

where $C$ is the relative average concentration and is a dimensionless value, $U$ is the average speed (unit: $\mathrm{m} / \mathrm{s}$ ), $K$ is the average turbulence kinetic energy $\left(\mathrm{m}^{2} / \mathrm{s}^{2}\right), \Phi$ is the average volume fraction, $u$ is the flow field velocity (unit: $\mathrm{m} / \mathrm{s}$ ), $z$ is the vertical height from water-sediment interface (unit: $\mathrm{m}$ ), $k$ is the turbulence kinetic energy $\left(\mathrm{m}^{2} / \mathrm{s}^{2}\right)$.

The vertical surface of the center of the overlying water in the geometric model is selected as the research object. The average velocity of the overlying water during the sediment starting is shown in Fig. 2. The average velocities of Case $1-6$ are $0.03 \mathrm{~m} / \mathrm{s}$, $0.08 \mathrm{~m} / \mathrm{s}, 0.13 \mathrm{~m} / \mathrm{s}, 0.23 \mathrm{~m} / \mathrm{s}, 0.35 \mathrm{~m} / \mathrm{s}$ and $0.50 \mathrm{~m} / \mathrm{s}$, respectively. In the initial stage of sediment starting, the average velocity of different cases is very small. With the increase in time, the average velocity of water increases rapidly and reaches a stable speed in a short time.

The vertical distribution of particle volume fraction of sediment at different time is analyzed by using Case 3 and Case 4 data, as shown in Fig. 3. At the initial stage of sediment starting, the distribution of particle volume fraction is similar under different velocity conditions. With the increase in time, the particle volume fraction in overlying water increases rapidly and reaches the same stable value in $2 \mathrm{~min}$, which is consistent with the experimental phenomena. The vertical distribution of sediment particles varies in physical process due to their different properties. For fine sediment $\left(D_{50}=0.03 \mathrm{~mm}\right)$, the spatial structure of sediment is uniform. The balance between upward suspension and downward deposition of sediment leads 


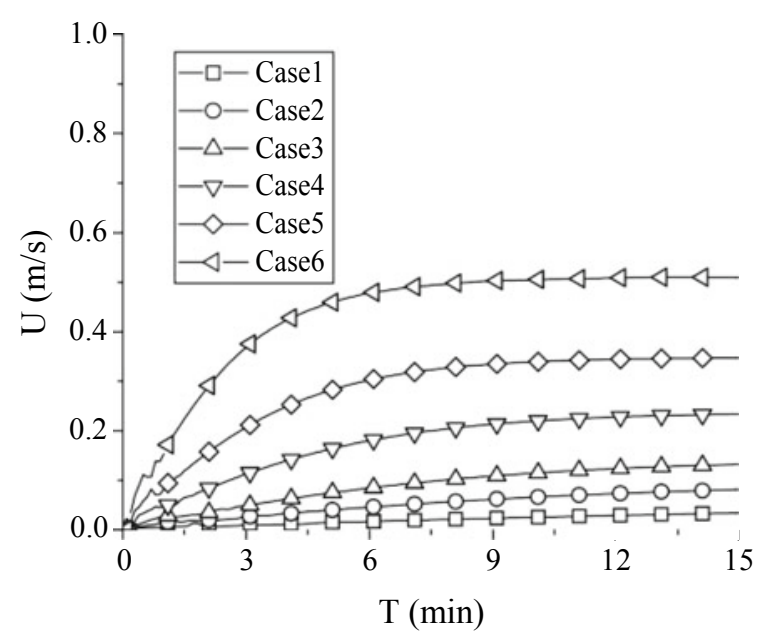

Fig. 2 Velocity changes with time during sediment starting

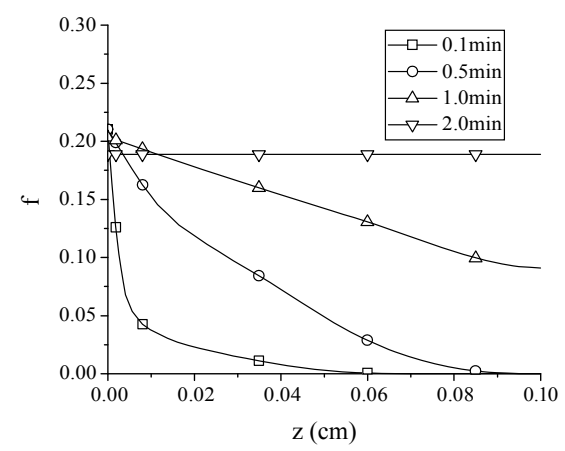

a) Case 3

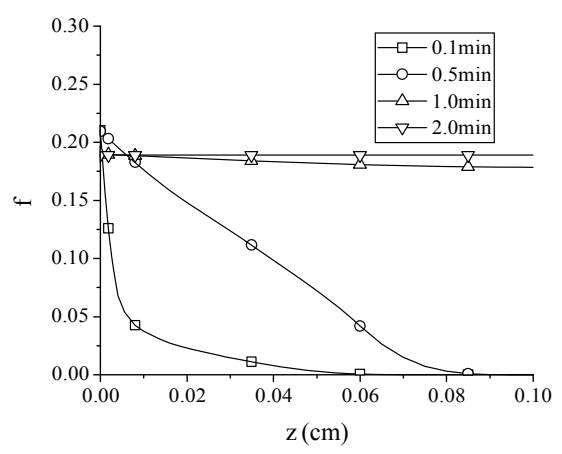

b) Case 4

Fig. 3 Vertical distribution of particle volume fraction during sediment starting at different times

to the distribution equilibrium of particle volume fraction. When the hydrodynamic conditions are constant, the equilibrium will not be broken. Considering that the deposition velocity of fine sediment is a function of volume fraction, the deposition velocity is smaller than that of coarse sediment, so the distribution of particle volume fraction along water depth is more uniform.

The sediment particles rapidly entering the overlying water have an impact on the flow characteristics of the overlying water and affect the release of contaminant. Data of Case 1, Case 3, Case 4 and Case 5 are selected to analyze the relationship between average turbulent kinetic energy, relative average concentration of contaminant and time at different velocity. Because of the small deposition velocity of fine sediment, the volume fraction of fine sediment quickly reaches equilibrium and remains stable. 
Contaminant in pore water also enters the overlying water rapidly and reachws the same equilibrium concentration soon. It is noteworthy that the average turbulent kinetic energy reaches its peak value after the starting of fine sediment, then decreases rapidly and gradually reaches a stable level over time. As the flow velocity increases, the turbulent kinetic energy increases, and the time for the contaminant to reach equilibrium decreases in the overlying water. During the process of contaminant equilibrium in the overlying water, convection and turbulent diffusion have made their respective contributions (Fig. 4).

When the adsorption and desorption of fine sediments are not considered, convective and turbulent diffusion have different effects on the process of contaminant balance in the overlying water. Analysis of the overlying water contaminant to reach the equilibrium, the relationship between the flux (total flux $N_{\mathrm{T}}$, convective flux $N_{\mathrm{C}}$, turbulent diffusion flux $N_{\mathrm{D}}$ ) and water flow characteristics (Reynolds number) is shown in Fig. 5. For non-adsorbed media, the total flux of the contaminant increases linearly with the velocity, that is, with the Reynolds number increasing linearly. When the Reynolds number is small $(0<\operatorname{Re}<35,000)$, the contribution of convection and turbulent diffusion is basically the same during the diffusion of contaminant. When the Reynolds number is large $(\operatorname{Re}>35,000)$, the contribution of turbulent diffusion decreases rapidly, and the contaminant diffusion is mainly dominated by convection.

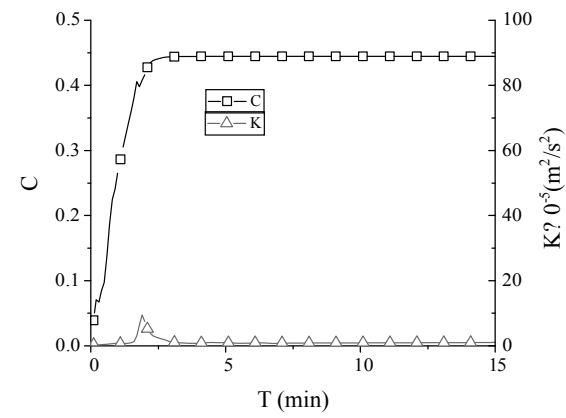

a) Case1

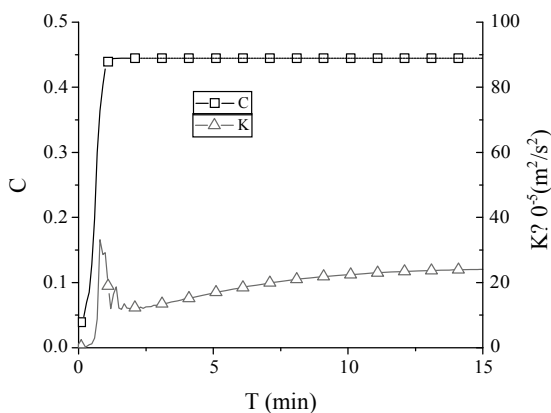

c) Case 4

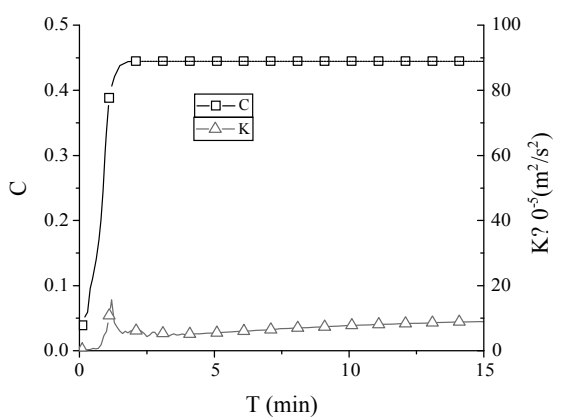

b) Case3

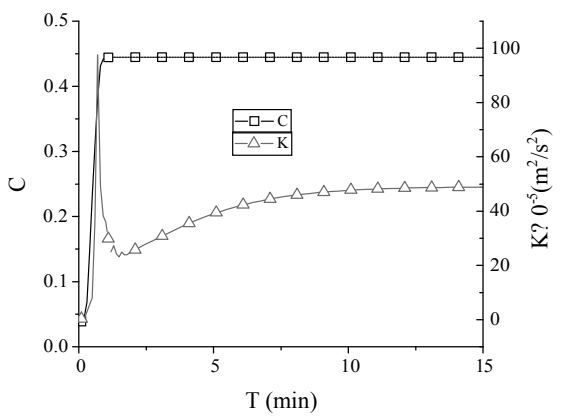

d) Case5

Fig. 4 The curve of average concentration, turbulent kinetic energy and time 


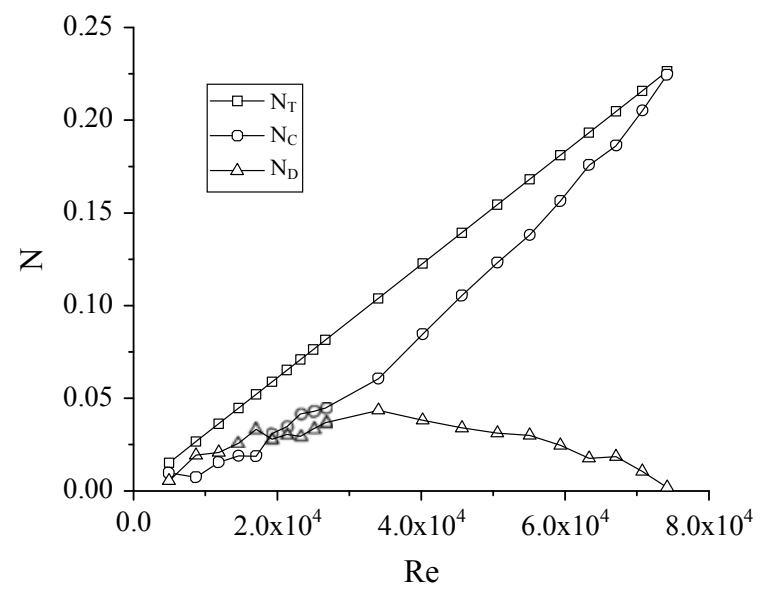

Fig. 5 Curve of contaminant release flux and Reynolds number

\section{Conclusions}

The re-suspension release process of sediment contaminant is a coupling process consisting of overlying water, sediment and contaminant. The complex flow field characteristics after sediment starting are the main influencing factors for the release of contaminant from sediment re-suspension. The fine sediment is susceptible to the effect of water flow. When re-suspension, the sediment particles volume fraction in overlying water reach equilibrium in short time $(<2 \mathrm{~min})$ and remain stable, because of uniform spatial structure and small deposition speed. The sediment particles rapidly entering the overlying water have an impact on the flow characteristics of the overlying water and affect the release of contaminant. The average turbulent kinetic energy reaches its peak value after the starting of fine sediment, then decreases rapidly and gradually reaches a stable level over time. As the flow velocity increases, the turbulent kinetic energy increases, and the time for the contaminant to reach equilibrium decreases in the overlying water. Convection and turbulent diffusion have different effects on the contaminant equilibrium process in the overlying water. When the Reynolds number is small $(0<\operatorname{Re}<35,000)$, the contribution of convection and turbulent diffusion is basically the same during the diffusion of contaminant. When the Reynolds number is large $(\operatorname{Re}>35,000)$, the turbulent diffusion contribution decreases rapidly, and the contaminant diffusion is mainly dominated by convection.

Acknowledgements The authors are grateful to the financial support by National Natural Science Foundation of China (No. 11602278, 11432015, 11872117 and 11802313), National Key R\&D Program of China (2018YFC1505504), and "Beijing Institute of Technology Research Fund Program for Young Scholars." 


\section{References}

1. Zhang C, Yu ZG, Zeng GM et al (2014) Effects of sediment geochemical properties on heavy metal bioavailability. Environ Int 73(4):270-281

2. Cheng P, Zhu H, Fan J, Fei M, Wang D (2013) Numerical research for contaminant release from un-suspended bottom sediment under different hydrodynamic conditions. J Hydrodynam 25(4):620-627

3. Cheng P, Zhu H, Zhong B, Fei M, Wang D (2014) Sediment rarefaction re-suspension and contaminant release under tidal currents. J Hydrodynam 26(5):827-834

4. Zhu HW, Cheng PD, Li W et al (2017) Empirical model for estimating vertical concentration profiles of re-suspended, sediment-associated contaminants. Acta Mech Sin 33(5):846-854

5. Stickel JJ, Powell RL (2005) Fluid mechanics and rheology of dense suspensions. Annu Rev Fluid Mech 37(1):129-149

6. Hinch EJ (2011) The measurement of suspension rheology. J Fluid Mech 686:1-4

7. Krieger IM, Dougherty TJ (1959) A mechanism for non-Newtonian flow in suspensions of rigid spheres. Trans Soc Rheol 3:137-152

8. Maron SH, Pierce PE (1956) Application of ree-eyring generalized flow theory to suspensions of spherical particles. J Colloid Sci 11(1):80-95

9. Quemada D (1977) Rheology of concentrated disperse systems and minimum energy dissipation principle. Rheol Acta 16(1):82-94

10. Mendoza CI, Santamari A-Holek I (2009) The rheology of hard sphere suspensions at arbitrary volume fractions: an improved differential viscosity model. J Chem Phys 130(4):044904

11. Shewan HM, Stokes JR (2015) Analytically predicting the viscosity of hard sphere suspensions from the particle size distribution. J Nonnewton Fluid Mech 222:72-81

12. Ignat $\mathrm{L}$, Pelletier $\mathrm{D}$, Ilinca $\mathrm{F}$ (2000) A universal formulation of two-equation models for adaptive computation of turbulent flows. Comput Methods Appl Mech Eng 189(4):1119-1139

13. Franca LP, Frey SL (1992) Stabilized finite element methods: II. The incompressible NavierStokes equations. Comput Methods Appl Mech Eng 99(2-3):209-233

14. Hughes TJR, Franca LP, Hulbert GM (1989) A new finite element formulation for computational fluid dynamics: VIII. The galerkin/least-squares method for advective-diffusive equations. Comput Methods Appl Mech Eng 73(2):173-189 